\title{
Feature Set Reduction for Source Camera Identification Using Demosaicing and Wavelet-based Features
}

\author{
Rishabh Sanghvi ${ }^{*}$, Laksh Hariyani² and Richa. R. Khandelwal ${ }^{3}$ \\ ${ }^{1},{ }^{2}$ Research Intern, Department of Electronics Engineering, Shri \\ Ramdeobaba College of Engineering and Management \\ ${ }^{3}$ Associate Professor, Department of Electronics Engineering, Shri \\ Ramdeobaba College of Engineering and Management, Nagpur, India.
}

\section{ABSTRACT}

Recent research work done in Camera Forensic Techniques have exhibited excellent results by merging several modern methods of Source Camera Identification, thereby creating a large feature set. Increasing the feature set can also contribute to higher computational time, complexity and error rates due to use of redundant features. The work exhibited in this paper shows that it is possible to optimize the feature set size to 16 , while maintaining the results as those obtained by state-of-the-art techniques. The statistical features extracted from images are based on demosaicing algorithm and wavelet transform. First, the proposed statistical equations were used to formulate 4 features based on periodic characteristics shown by demosaicing. Then, the image matrices were transformed by 1-Level wavelet decomposition and 16 features were extracted from obtained co-occurrence matrices. After further optimization, it was found that classification model trained using 16 features had the highest average accuracy of 99.2\%. Thus, the final optimized feature set size was reduced to 16 features from 20 features for identifying images from 10 camera models.

KEY WORDS: SOURCE CAMERA IDENTIFICATION (SCI), DEMOSAICING ALGORITHM, WAVELET FEATURES, HAAR AND SYMLET WAVELET, SUPPORT VECTOR MACHINE (SVM).

\section{INTRODUCTION}

Images are visual representation of information which is used for forensic, educational, entertainment and research purposes. Some individuals post fake information by manipulating images for gaining popularity or money. Source Camera Identification (SCI) is one such technique which will help us to identify the source of such suspected images and to quantify the fake and real information. [Kaur. H. Jindal, 2020] briefly explains the scope and

\section{ARTICLE INFORMATION}

*Corresponding Author: sanghvirh@rknec.edu

Received 18th Oct 2020 Accepted after revision 27th Dec 2020

Print ISSN: 0974-6455 Online ISSN: 2321-4007 CODEN: BBRCBA

Thomson Reuters ISI Web of Science Clarivate Analytics USA and Crossref Indexed Journal

\section{Clarivate
Analytics}

NAAS Journal Score 2020 (4.31)

A Society of Science and Nature Publication,

Bhopal India 2020. All rights reserved.

Online Contents Available at: http//www.bbrc.in/

Doi: http://dx.doi.org/10.21786/bbrc/13.14/47 applications of image and video forensics. The proposed methodology is a step towards providing desired solution to the existing problem. Several approaches are available for SCI like study of Color Features, Demosaicing Algorithm, Gray-Level Co-occurrence Matrix (GLCM), Wavelet transform, Image Quality Metrics (IQM), Noisebased techniques and many others, shown by [M. Kharrazi, 2004]. Such methods are constructed after studying the unique features of camera itself (proprietary hardware and software). Such techniques are broadly classified as Image Feature Extraction Techniques.

One important camera hardware is the Color Filter Array (CFA) or Bayer Filter, shown in Fig. 1. It captures all color components simultaneously, thus we need demosaicing algorithm for calculating missing color intensities to form a color image. The process of demosaicing approximates pixel intensity for all color channels based on the neighbouring pixels. There are several different algorithms for demosaicing which take under 
consideration various sizes of kernel for approximation of pixel intensities. Many such algorithms are briefly explained by [Y. Long, 2006].

Figure 1: Bayer Pattern (Color Filter Array)

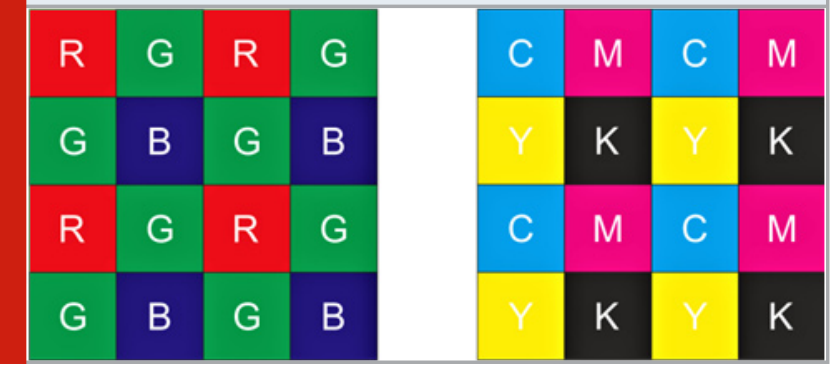

Several approaches have been shown in different research works for using Demosaicing Algorithm as SCI technique. [A. C. Gallagher, 2005], proposed the use of second order differential equation to identify traces of interpolation occurring in images due to demosaicing. An improvement to Gallaghar's method was proposed by [S. Bayram, 2005], [S. Bayram, 2006]. First, they segmented the image based on smoothness, then the second order differential equation method was applied to smooth parts and Expectation- Maximization (EM) algorithm to less smooth parts of the image. [J. S. Ho, 2010] constructed a method to extract the correlations from the images, for constant hue interpolation based demosaicing algorithm. A novel framework was proposed by [Chen Chen, 2015], which calculates distribution of demosaicing errors over the entire image, which is represented as a co-occurrence matrix. Another framework developed by [H. Cao, 2009], accurately detects demosaicing regularities in images. First the framework reverse classifies images to several categories and then estimates the Demosaicing Formula based on partial second order differential equation. All these techniques finally extract features from the matrices obtained and then identify source of the images using Machine Learning (ML) Algorithms.

The images can also be transformed and depicted into different domains using Fourier, Wavelet, Cosine and many other transforms. Transformations ease the process of feature extraction as different transforms reveal different characteristics of camera's processing steps that are hidden in the images. Hence, Wavelet Transform are widely used for SCI and also Image Processing. Also, Wavelet transform is found to compliment many techniques, for e.g. noise-based techniques and Gray Level Co-occurrence Matrix (GLCM) based features, and ends up enhancing the performance of classification model. [B. Wang, 2009] suggested use of 4-Scale wavelet decomposition for extracting 216 higher order wavelet features and 135 wavelet co-occurrence features.

An extension of the above work was done by [A. Jeyalakshmi, 2018], where the effectiveness of using different types of wavelet transform for feature extraction was compared. An approach carried out by [K. R. Akshatha, 2016] used Higher Order Wavelet Statistics (HOWS) for extracting PRNU noise. The wavelet denoising techniques were utilized for extracting noise from the images. The rest of the paper is distributed as follows: Section 2 contains Proposed Methodology and a novel approach of using available techniques with optimum number of features will be discussed. Section 3 defines the Experimental Setup, the number of camera models used for testing proposed methodology. Section 4 contains the Experimental Results obtained. In Section 5 the advantages, limitations of proposed methodology and future work are discussed.

2. Proposed Methodology: Improvements and modifications to currently available methods for demosaicing and wavelet-based techniques are discussed. These improvements helped to reduce the number of features in comparison to available techniques, while maintaining a low error rate of classification accuracy.

\subsection{Proposed Statistical Features based on Demosaicing} methods: Gallaghar's method has certain limitations, its performance reduces significantly after image compression (e.g. standard JPEG compression). It becomes difficult to identify peaks, as for some observations they are indifferentiable from false peaks occurring due to JPEG compression at defined intervals. Also, the highest peak tends to shift around in the spectrum for images taken from same camera model, due to which false classification of images to other source camera model increases. The above techniques concentrate on a single peak (the highest peak which reveals the interpolation factor) from the entire frequency response of pseudo-variance signal. Another approach is to formulate features after studying the entire signal, which would give an insight about the shape of the signal and distribution of peaks. Hence, we propose two statistical features accompanied by skewness and kurtosis features, that would be able to extract more information from the entire spectrum of pseudo-variance signal. This in turn would provide a desired classification accuracy comparable with state-of-the-art methods and optimize the overall feature set.

The proposed method can be applied on any one of the color channel of the image (either Red, Blue or Green Color Channel). We selected the Red Color Channel, but the result can be replicated if one uses any of the color's matrix (which contains pixel intensity of selected color). First, we calculated the second order differential for each row of the image (from the equation provided by Gallaghar et al), added the result column-wise and averaged over the total number of columns. Then we converted the signal into its frequency equivalent using Discrete Fourier Transform (DFT). The methodology is exactly similar to Gallaghar et al's till this point. Then proposed statistical formulae are employed to extract the following features:

\section{a. Coefficient Periodicity Analysis Feature (CPAF)}

$$
C P A F=\frac{1}{C} \sum_{i=1}^{C} F(1, i)
$$




\section{b. Peak Distribution Analysis Feature (PDAF)}

$P D A F=\frac{1}{C} \sum_{i=1}^{C} i * F(1, i)$

Figure 2: The differences in peak magnitudes, their distribution and shape of signal are shown for 4 camera models. All camera models captured exactly the same scenery.
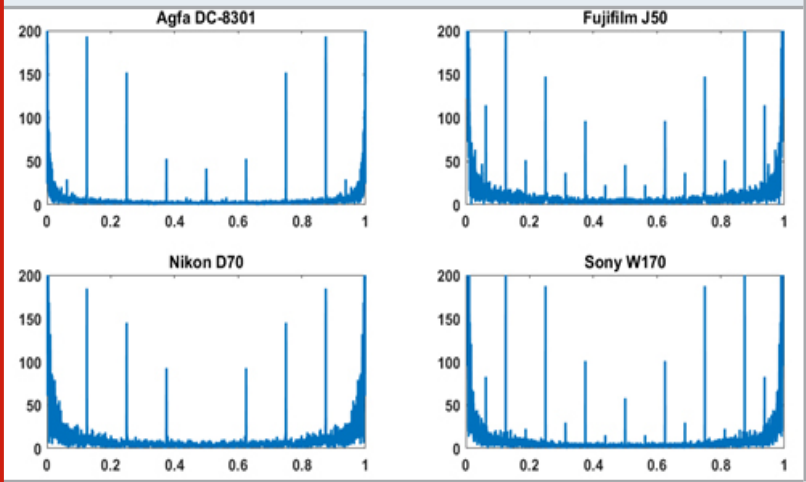

$C$ represents the no. of columns in the image matrix and $\mathrm{F}(1, \mathrm{i})$ is the magnitude at ith column of the frequency signal.(The value of $\mathrm{C}$ is used to reduce the numerical values of the features, it doesn't affect the performance, as the features extracted without using $C$ yield the same results). As every camera uses a different demosaicing algorithm, the distribution and values of the weighted coefficients will be different. Therefore, the peaks will have different magnitudes(as depicted in Fig. 2.) as periodicity of the coefficients will also differ. The feature-1 calculates a value by adding all the peak magnitudes. The sum will thus depend on the value of the coefficients and their periodicity in the variance signal, (hence, referred as CPAF) which will be similar for images from same camera model taken into consideration, as the coefficient is mainly affected by the type of demosaicing algorithm or size of kernel used.

Another important data to be extracted, for efficiently using the CPAF is distribution of peaks in the variance signal. As CPAF is a sum of magnitudes, the results obtained for some images from different cameras can be same, but the distribution of peaks will be different. The distribution of the peak values can then help us to determine the camera model (hence, referred as PDAF). The PDAF is a sum of product of peak magnitudes and their respective position. The sum will vary depending upon the position at which the peak magnitudes occur.

2.2. Skewness and Kurtosis: These statistical features were used to further analyze the shape and distribution of data with respect to the mean of the signal. These 2 features were added to further improve the performance. The results observed validated there use, as the error rates reduced significantly.

3. Wavelet-based Feature Extraction: The proposed methodology uses 1-Scale Wavelet decomposition and then obtain Energy, Entropy, Homogeneity and Contrast features from the co-occurrence matrices. The 1-level wavelet transformation can provide a set of uncorrelated features with minimum decomposition of the signal. Hence, we propose the use of 1-Scale Wavelet decomposition in addition to the proposed statistical features for classifying images from multiple cameras and to improve the results obtained from latter technique. These features were extracted using 3 different wavelet types, for comparing their individual performance and to incorporate the best performing wavelet's features with demosaicing based features. The three families of wavelet included Haar, Coiflet \& Symlet wavelet.

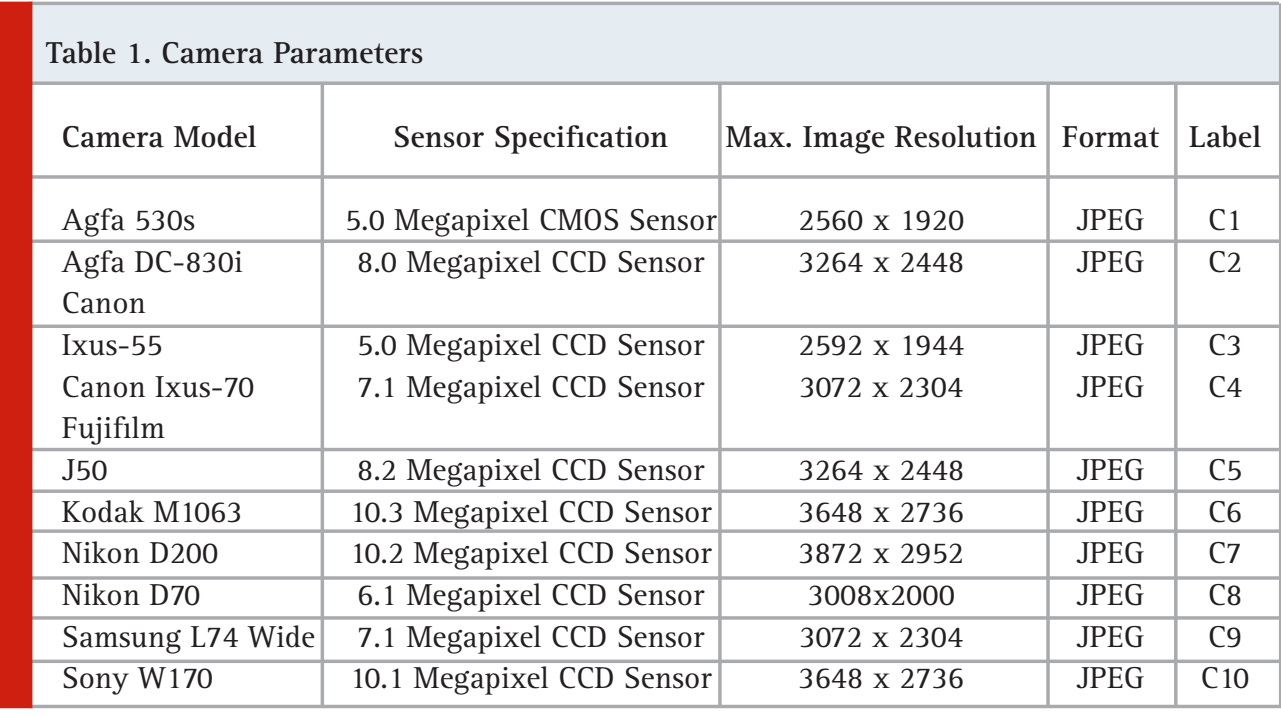

4. Experimental Setup: To observe the effectiveness and performance of proposed features to identify source camera model, 2924 images were collected from 10 camera models (300 images from 9 camera and 224 from Cannon Ixus-55 camera, this is maximum images available for this camera) from Dresden Image Database [Gloe, T., \& Böhme, 2010], which is a standard database used for benchmarking of camera-based forensic techniques. 
The specifications of the source camera models used for training and testing purposes are given in Table I. Hence, the database contains images that are from different brands, same camera brand but different model and some having the same image resolution.

Figure 3: Feature Set Extraction Setup

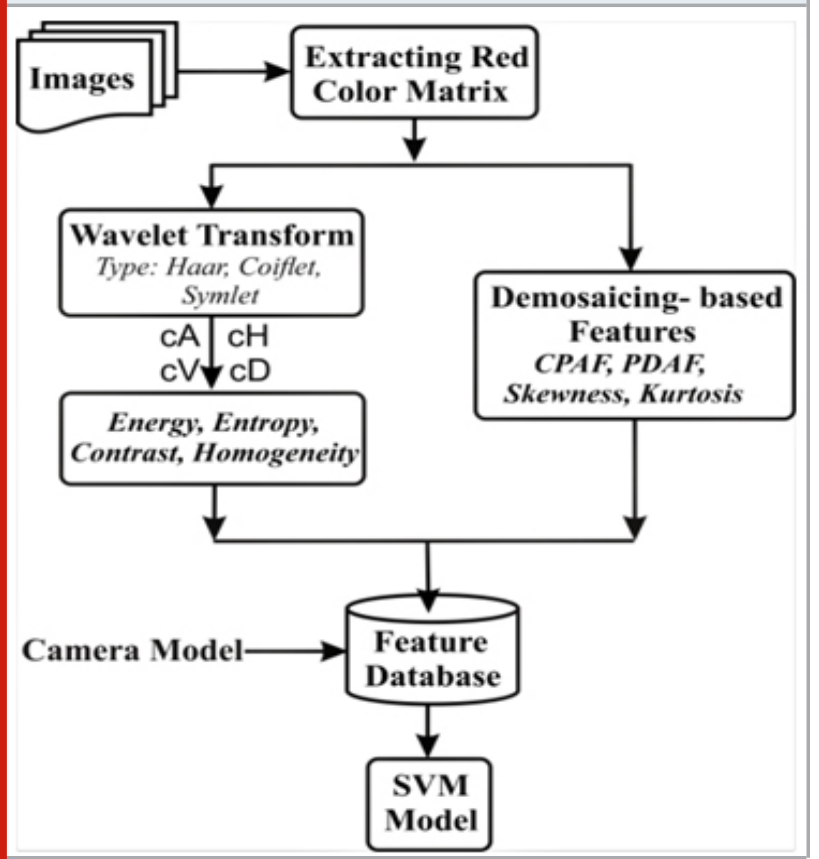

The 4(statistical features from demosaicing) + [4(cA, cV, cH, cD) x 4(Energy, Entropy, Homogeneity, Contrast)] $=20$ features were calculated for a single image. This process of full feature set extraction from images is depicted in Fig. 3. For measuring the performance of the classification learner, the camera model name associated with each image is fed into the classifier along with the feature set. After collecting features from each image, a SVM classifier was used for training. We tested the model by applying 5-fold Cross-Validation. The results were verified by applying Holdout Validation, in which we split the data into $80 \%$ - 20\%, training - test set. The process is repeated for Haar, Coiflet \&t Symlet wavelet.

\section{EXPERIMENTAL RESULTS}

\section{The experiments mentioned below were performed on MATLAB.}

\subsection{Performance of Demosaicing based Features: The} features were tested on different pairs of camera models mentioned in Table III. The camera models included in 3 sets contain camera models of same brand, as many techniques of SCI show poor performance when camera from same brands are incorporated in the sets. The results obtained are then compared with other feature based techniques on the basis of feature set size used and obtained accuracy in Fig. 4. The proposed methodology achieved higher classification accuracy by using the least amount of features among all given methods. The accuracy does vary depending upon the similarity in demosaicing algorithm used in the cameras, shown in Fig. 4.

The set $\mathrm{C}$ has a lower accuracy and this indicates that the cameras use similar demosaicing methods. As the extracted features are dependent on demosaicing algorithm, such instances can be avoided by adding features based on other methodologies to the feature set. When further tested on 10 cameras, results showed that the confusion was most evident for camera pairs using similar demosaicing algorithms, as also indicated by Bayram et al. Thus, it can be concluded that other methods are required, different from those based on demosaicing algorithm, to achieve better results for multiple source camera models. Thus, we selected wavelet-based features to differentiate between such camera models.

Figure 4: The accuracy obtained by proposed and referenced methodologies are depicted and compared. The brackets contain the feature set size of respective methodologies.

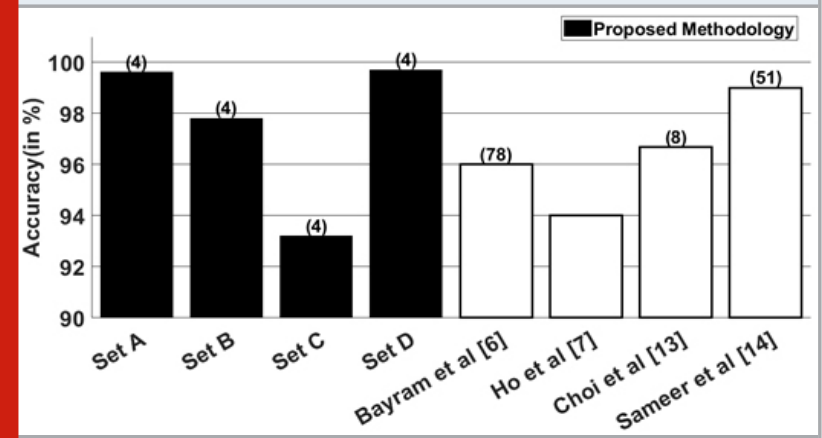

5.2. Performance of Full Feature Set (Demosaicing and Wavelet Features): The feature set initially consisted of 4 features from demosaicing, then features like Entropy and Contrast (as Energy and Homogeneity were found to have similar values for images with same resolution. Hence, to remove the dependency of feature set on original size of image, these features were removed) extracted using Haar and Symlet were added successively to build a set of 20 features as discussed earlier. Initially, the set of 4 demosaicing features was tested on 10 camera models, its performance was analyzed and then 4 features obtained from wavelet features(i,e, Entropy and Contrast) were added successively. The accuracy obtained after each iteration is depicted in Fig. 5. This experiment clearly shows how the wavelet features improved the overall performance of the system (see transition from 4 to 12 features) and the contribution of each wavelet type in maximizing the performance (see transition from 12 to 16 features).

The important observation made was, the SVM model has the highest accuracy among the 4 training models for 16 features. As, the performance of the model is same for 16 and 20 feature set size, evident from the straight line between them in Fig. 5, we kept 16 features in the final set. The final feature set thus consisted of 4 (statistical features from demosaicing) $+[2$ (entropy + 
contrast) $\mathrm{x} 4(\mathrm{cA}, \mathrm{cH}, \mathrm{cV}, \mathrm{cD})]$ from Haar + [1(entropy) $\mathrm{x} 4(\mathrm{cA}, \mathrm{cH}, \mathrm{cV}, \mathrm{cD})]$ from Symlet $=16$ features, that are applied to 2924 images collected from 10 camera models. In another test, the optimized feature set was first used to classify images from 2 camera models and then a new camera model was introduced in every iteration. The average accuracy obtained for every set is shown in Fig. 6. This experiment allowed proper comparison of proposed methodology with research studies done for SCI, which use different number of camera models. It was also observed that the error rates rose utmost by $0.2 \%$, every time a new camera was added. The figure also shows that the proposed methodology has better or on par classification accuracy when compared with referenced methodologies, after using the least amount of features. The average accuracy of SVM classifier was $99.2 \%$

Figure 5: Comparing classification accuracy of different training algorithms.

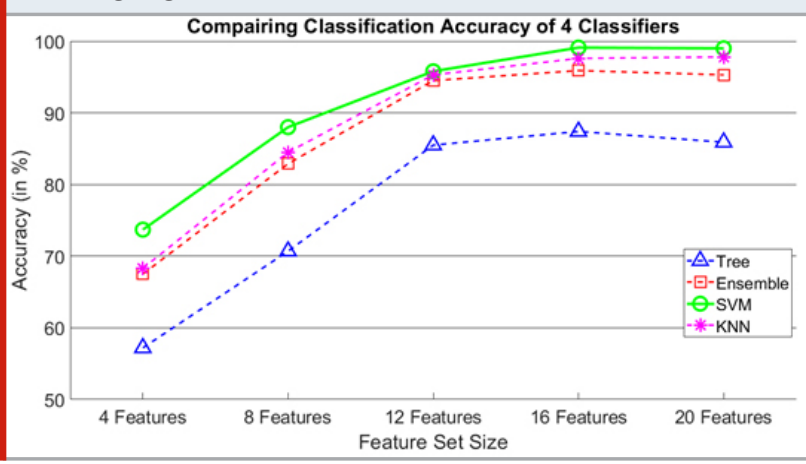

Figure 6: Comparing performance of proposed methodology with available SCI techniques based on camera models used vs. accuracy obtained. The brackets indicate full feature set size.

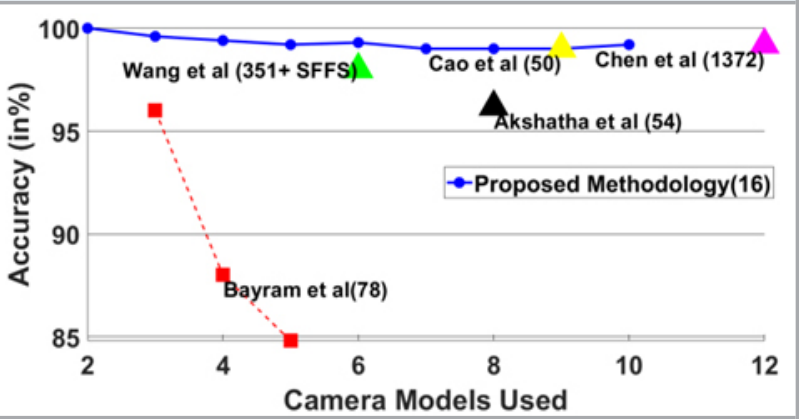

Table 3. Camera Set Contents

\begin{tabular}{|c|c|}
\hline Set & Camera Models included \\
\hline Set A & Canon Ixus-70, Kodak \\
& M1063, Nikon D70 \\
\hline Set B & Agfa DC-530s, Agfa DC-8301, \\
& Canon Ixus-70 \\
\hline Set C & Nikon D70, Nikon \\
& D200, Samsung L74 \\
\hline Set D & Nikon D200, Canon Ixus-70, Sony \\
& W170, Canon Ixus-55 \\
& (similar set used by Ho et al) \\
\hline
\end{tabular}

Figure 3: UV-VIS Spectra of the compound, PA-B-ester

\begin{tabular}{|c|c|c|c|c|c|c|c|c|c|c|c|}
\hline \multirow{2}{*}{$\begin{array}{l}\text { Actual } \\
\text { Class }\end{array}$} & \multicolumn{2}{|l|}{ Predicted Class } & \multicolumn{1}{l|}{ Accuracy } \\
\cline { 2 - 12 } & C1 & C2 & C3 & C4 & C5 & C6 & C7 & C8 & C9 & C10 & \\
\hline C1 & 298 & & & & & & 1 & 1 & & & $99 \%$ \\
\hline C2 & & 297 & & 1 & & 1 & & & 1 & & $99 \%$ \\
\hline C3 & & & 223 & 1 & & & & & & & $99 \%$ \\
\hline C4 & & 1 & & 299 & & & & & & & $99 \%$ \\
\hline C5 & & & & & 300 & & & & & & $100 \%$ \\
\hline C6 & & & & & & 296 & & & & 4 & $99 \%$ \\
\hline C7 & & 1 & & & 1 & 1 & 297 & & & & $99 \%$ \\
\hline C8 & 6 & & & & & & & 294 & & & $98 \%$ \\
\hline C9 & & & & 3 & & & & & 297 & & $99 \%$ \\
\hline C10 & & & & & & & & & & 300 & $100 \%$ \\
\hline
\end{tabular}

(Cross-Validation accuracy), shown in Table IV. Also, the Holdout Validation accuracy was $99.7 \%$ for the test set, obtained after $80 \%$ training set - 20\% test set division of the entire database of 2924 images. Thus, the number of features was reduced to 16, without affecting the classifier's accuracy.

\section{CONCLUSION AND FUTURE WORK}

The proposed methodology can successfully identify camera models with high precision using only 16 features which is less than many methodologies available at present. The features were extracted based on periodic characteristics of demosaicing and by transforming 
images by wavelet transform. The classification model maintains a low error rate when a new source camera is introduced, but the error rates will rise if multiple camera models (e.g. 10 cameras) are added, as the probability of false classification would increase. Thus, we need adaptive algorithms (like SFFS) that would be able to detect rise in false classification and then add uncorrelated features extracted from other wavelet families like Daubechies, Morlet, Shannon and many more to the feature set. The testing of these wavelet families is necessary before incorporating them with proposed methodology. In our future work, we would like to compare the effectiveness of using different wavelet families in addition with our proposed feature set.

\section{REFERENCES}

A. C. Gallagher, "Detection of linear and cubic interpolation in JPEG compressed images", Presented at CRV'05, Victoria, BC, Canada, 2005, pp. 65-72.

A. Jeyalakshmi and D. R. Chitra, "Comparative study of feature extraction using several wavelet transforms for source camera identification," 2018 2nd International Conference on Inventive Systems and Control (ICISC), Coimbatore, 2018, pp. 1407-1414.

B. Wang, Y. Guo, X. Kong and F. Meng, "Source Camera Identification Forensics Based on Wavelet Features," 2009 Fifth International Conference on Intelligent Information Hiding and Multimedia Signal

Processing, Kyoto, 2009, pp.702-705.

Chen Chen and M. C. Stamm, "Camera model identification framework using an ensemble of demosaicing features," 2015 IEEE International Workshop on Information Forensics and Security (WIFS), Rome, 2015, pp.1-6, doi:10.1109/WIFS.2015.7368573.

Gloe, T., \& Böhme, R. (2010). The 'Dresden Image Database' for benchmarking digital image forensics. In Proceedings of the 25th Symposium on Applied Computing (ACM SAC 2010), Vol. 2, pp.1585-1591.

H. Cao and A. C. Kot, "Accurate Detection of Demosaicing Regularity for Digital Image Forensics," in IEEE Transactions on Information Forensics and Security, vol. 4, no. 4, pp. 899-910, Dec. 2009, doi:
10.1109/TIFS.2009.2033749.

J. S. Ho, O. C. Au, J. Zhou and Y. Guo, "Inter-channel demosaicking traces for digital image forensics," 2010 IEEE International Conference on Multimedia and Expo, Suntec City, 2010, pp. 1475-1480, doi: 10.1109/ ICME.2010.5582951.

Kaur H. Jindal, N. Image and Video Forensics: A Critical Survey. Wireless Pers Commun 112, 1281-1302 (2020). https://doi.org/10.1007/s11277-020-07102-X.

K.R. Akshatha, A.K. Karunakar, H. Anitha, U. Raghavendra, Dinesh Shetty, "Digital camera identifification using PRNU: A feature based Approach" in Digital Investigation, Vol. 19, pp. 69-77, December 2016.

K. S. Choi, E. Y. Lam and K. K. Y. Wong, "Feature Selection in Source Camera Identification," 2006 IEEE International Conference on Systems, Man and Cybernetics, Taipei, 2006, pp. 3176-3180, doi: 10.1109/ ICSMC.2006.384605.

M. Kharrazi, H. T. Sencar and N. Memon, "Blind source camera identification," 2004 International Conference on Image Processing, 2004. ICIP '04., Singapore, 2004, pp. 709-712 Vol. 1.

S. Bayram, H. Sencar, N. Memon and I. Avcibas, "Source camera identification based on CFA interpolation," IEEE International Conference on Image Processing 2005, Genova, 2005, pp. III-69.

S. Bayram, H.T. Sencar, and N. Memon, "Improvements on source camera model identification based on CFA Interpolation.”, In IFIP WG 11.9 International Conference on Digital Forensics, Orlando, FL, 2006.

V. U. Sameer, A. Sarkar and R. Naskar, ”Source camera identification model: Classifier learning, role of learning curves and their interpretation," 2017 International Conference on Wireless Communications, Signal Processing and Networking (WiSPNET), Chennai, 2017, pp. 2660-2666, doi: 10.1109/WiSPNET.2017.8300246. Y. Long and Y. Huang, "Image Based Source Camera Identification using Demosaicking," 2006 IEEE Workshop on Multimedia Signal Processing, Victoria, BC, 2006, pp. 419-424. 